Tropical Journal of Pharmaceutical Research January 2021; 20 (1): 191-195

ISSN: $1596-5996$ (print); 1596-9827 (electronic) (C) Pharmacotherapy Group, Faculty of Pharmacy, University of Benin, Benin City, 300001 Nigeria.

\title{
Utilization of adjusted body weight for dosing unfractionated heparin in obese patients with venous thromboembolism: A retrospective matched cohort study
}

\author{
Mohammed Alessa ${ }^{1}$, Jawaher Gramish ${ }^{2}$, Hind Almodaimegh ${ }^{2}$, Moteb A \\ Khobrani ${ }^{3}$, Lori Hornsby ${ }^{4}$, Abdullah A Alhifany ${ }^{5 *}$ \\ ${ }^{1}$ Clinical Pharmacy Department, College of Pharmacy, King Saud University, ${ }^{2}$ College of Pharmacy, King Saud bin Abdulaziz \\ University for Health Sciences, Riyadh, ${ }^{3}$ Clinical Pharmacy Department, College of Pharmacy, King Khaled University, Abha, \\ Saudi Arabia, ${ }^{4}$ Columbus Regional Health, Midtown Medical Center, Columbus, GA, USA, ${ }^{5}$ Clinical Pharmacy Department, \\ College of Pharmacy, Umm Al-Qura University, Makkah, Saudi Arabia
}

*For correspondence: Email: aahifany@uqu.edu.sa; Tel: +966-555521820

Sent for review: 18 July 2020

Revised accepted: 22 December 2020

\begin{abstract}
Purpose: To evaluate the effectiveness of adjusted body weight (AjBW)-based dosing of unfractionated heparin (UFH) in obese patients vis a vis actual body weight (ABW)-based dosing in non-obese patients with venous thromboembolism (VTE).

Methods: A retrospective chart review was conducted for obese and non-obese patients initiated on UFH for treating VTE from September 2013 to December 2014. Patients were excluded from the study if they were under 18 years old, developed heparin-induced thrombocytopenia during treatment, received thrombolytic therapy prior to UFH, or received UFH at a dose that did not follow the institution's protocol. The primary objective was to assess the efficacy of dosing UFH based on AjBW in achieving a therapeutic activated partial thromboplastin time (aPTT) within the first $24 \mathrm{~h}$ in obese patients, in comparison to the standard ABW-dosing for non-obese.

Results: Of the 57 patients included in the study, 27 patients (47.4\%) were obese, and 30 patients (52.6\%) were non-obese; 16 (59.25\%) of the obese patients achieved a therapeutic aPTT within the first $24 \mathrm{~h}$ of AjBW-based dosed UFH, while 18 (60\%) of the non-obese patients achieved a therapeutic aPTT within the first $24 h$ of ABW-based dosed UFH $(p=0.45)$.

Conclusion: $A j B W$-based dosing of UFH in obese patients demonstrates comparable efficacy to $A B W$ based dosing in non-obese patients.
\end{abstract}

Keywords: Obesity, Unfractionated heparin, Venous thromboembolism, Adjusted body weight, Ideal body weight

\begin{abstract}
This is an Open Access article that uses a fund-ing model which does not charge readers or their institutions for access and distributed under the terms of the Creative Commons Attribution License (http://creativecommons.org/licenses/by/4.0) and the Budapest Open Access Initiative (http://www.budapestopenaccessinitiative.org/read), which permit unrestricted use, distribution, and reproduction in any medium, provided the original work is properly credited.

Tropical Journal of Pharmaceutical Research is indexed by Science Citation Index (SciSearch), Scopus, International Pharmaceutical Abstract, Chemical Abstracts, Embase, Index Copernicus, EBSCO, African Index Medicus, JournalSeek, Journal Citation Reports/Science Edition, Directory of Open Access Journals (DOAJ), African Journal Online, Bioline International, Open-J-Gate and Pharmacy Abstracts
\end{abstract}

\section{INTRODUCTION}

Obesity is a common public health problem in the U.S. despite federal and local containment initiatives. In 2016, the prevalence of obesity in U.S. was reported to be $39.8 \%$ in adults and $18.5 \%$ in youth [1]. A major, independent risk factor of obesity is venous thromboembolism 
(VTE) [2].

VTE is primarily treated by unfractionated heparin (UFH) that requires actual body weight (ABW)-based dosing to achieve therapeutic activated partial thromboplastin time (aPTT) within 24 hours of starting the UFH infusion per The American College of Chest Physicians (ACCP) guidelines; however, there is a paucity of literature regarding the dosing of UFH in obese patients [3-8]. Few studies introduced adjusted body weight (AjBW)-based dosing of UFH in obese patients due to the concern of excessive anticoagulation and bleeding risk with ABWdosing $[9,10]$. Nevertheless, underdosing in this population is also a major concern. Therefore, the most appropriate dosing strategy in these individuals remains a topic of discussion $[11,12]$.

Hence, the main objective of this study is to evaluate the effectiveness of AjBW-based dosing of UFH in obese patients versus $A B W$-based dosing in non-obese patients with VTE.

\section{METHODS}

A retrospective chart review was conducted for patients who received intravenous infusion (IVF) of UFH for the treatment of VTE from September 2013 to December 2014. Obese and non-obese patients aged 18 years and older and admitted with acute VTE were included in the study. Obesity was defined as an ABW greater than $130 \%$ of ideal body weight (IBW). UFH dosing for VTE treatment followed an internal protocol at which a loading dose of 80 units per kilogram $(\mathrm{kg})$ of body-weight is followed by an initial infusion rate of 15 - 18 units/kg per hour (h) to achieve a target aPTT of 74 - 106. ABW is utilized for dosing in non-obese patients, while $\mathrm{AjBW}$ was calculated using Eq 1.

$A j B W=\{I B W+0.3(A B W-I B W)\}$

This has been utilized for dosing UFH in obese patients. IBW was calculated as in Eq 2 [13].

$\mathrm{IBW}=\{45.4+0.89 \times$ (height $(\mathrm{cm})-152.4)+4.5$ (if female) $\}$

Patients were excluded from the study if they were under 18 years of age, developed heparininduced thrombocytopenia (HIT) during treatment, received thrombolytic therapy prior to UFH initiation, or received UFH that did not follow the above-mentioned institution's protocol.

Ethical approval has been obtained from the Auburn University Institutional Review Board (IRB) (approval no. 15-014 EP 1502) and the
Columbus Regional Integrity Panel. (Appendix A) In addition, the STROBE guidelines were followed in this study [14]. Furthermore, the following data were collected from the included patients' electronic medical records: basic demographic information such as age, gender, height and weight; VTE diagnosis, length of stay; heparin dosing information such as time of initiation of UFH, initial loading dose and infusion rate; baseline and subsequent aPTT values and time to first therapeutic aPTT; any supra therapeutic aPTT values; number of unfractionated heparin dose adjustments required to achieve a therapeutic aPTT; and any bleeding or thrombotic events.

The primary outcome was to compare the percentage of subjects who achieved a therapeutic aPTT within the first $24 \mathrm{~h}$ between the obese patients who received UFH based on AjBW and non-obese patients who received UFH based on ABW. The secondary outcome was to compare both groups in terms of the time required to achieve a therapeutic aPTT, the number of supratherapeutic aPTT values, the average number of UFH dose adjustments required to achieve a therapeutic aPTT, and the number of bleeding or thrombotic events. The primary safety outcome was clinically significant bleeding, including both major and non-major bleeding that required UFH discontinuation [15].

\section{Statistical analysis}

Statistical analyses were performed via an independent Student's $t$-test for continuous variables and $x^{2}$ test for categorical data. The $p$ value was considered significant if $<0.05$. Statistical analysis was done by using IBM SPSS Statistics for Windows, Version 23.0 (Armonk, NY: IBM Corp).

\section{RESULTS}

Overall, 600 patient charts were reviewed, and 540 patients were excluded because they received UFH for cardiac indications $(n=543)$ or not according to the hospital's protocol $(n=5)$ or developed HIT during treatment $(n=4)$. The remaining 57 patients were included in the study. In those 57 patients, 27 patients (47.4\%) were obese, and 30 patients (52.6\%) were non-obese (Table 1).

The mean initial bolus dose of UFH was $79.07 \pm$ 5.753 and $79.44 \pm 5.154 \mathrm{unit} / \mathrm{kg}$ in the obese and non-obese patients, respectively. Similarly, the mean initial infusion dose was found to be 14.59 \pm 1.474 and $14.40 \pm 1.714 \mathrm{units} / \mathrm{kg} / \mathrm{h}$ in obese and non-obese patients, respectively. Figure 1 
shows that out of 27 subjects, 16 (59\%) obese patients who received AjBW-based dosing achieved a therapeutic aPTT within the first $24 \mathrm{~h}$ of UFH initiation. Similarly, $18(60 \%)$ out of 30 non-obese patients who received ABW-based dosing achieved a therapeutic aPTT within the first $24 \mathrm{~h}$ of UFH initiation ( $p=0.45)$. Moreover, $26 \%$ of obese patients achieved a therapeutic aPTT within > 24 to $48 \mathrm{~h}$ versus $27 \%$ of nonobese patients $(p=0.59)$. Furthermore, the remaining $15 \%$ of obese patients achieved therapeutic aPTT after $48 \mathrm{~h}$ as compared to $13 \%$ of non-obese patients $(p=0.46)$.

Table 1: Demographic characteristics of participants' population

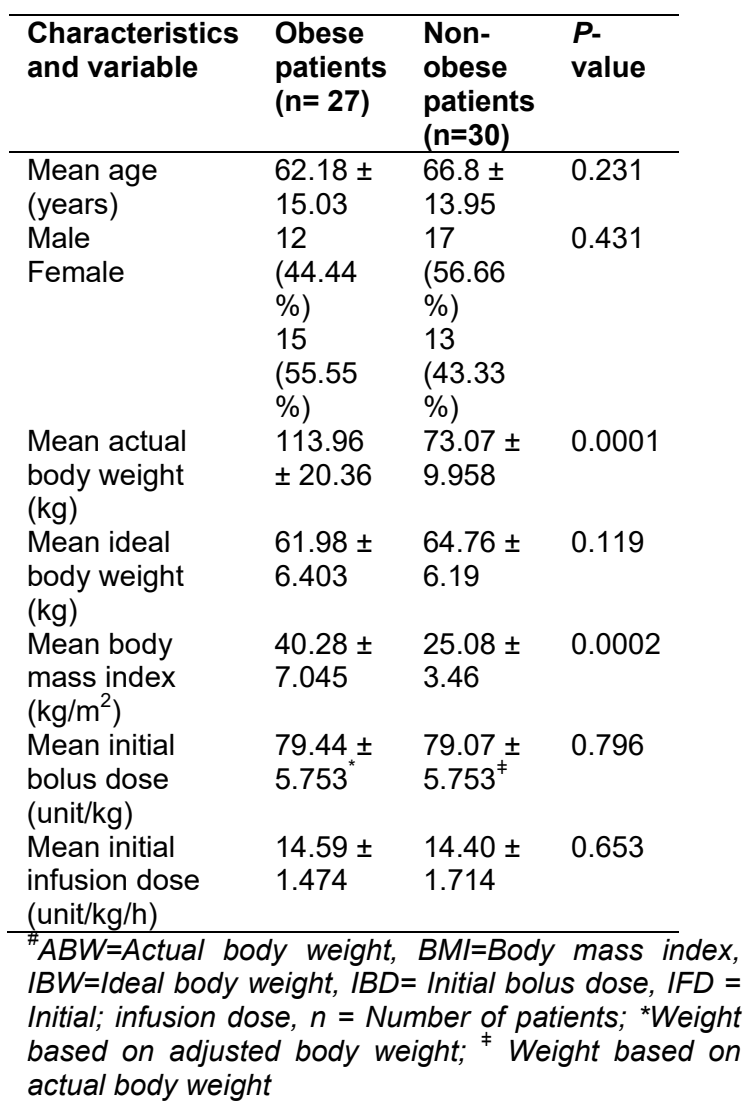

Among the obese patients not achieving a therapeutic aPTT within the first 24 hours, 19\% had sub-therapeutic aPTT values and $22 \%$ had supratherapeutic aPTT values. Similarly, in the non-obese group, $17 \%$ had sub-therapeutic aPTT values while $23 \%$ had supratherapeutic aPTT values. Throughout the entire treatment period, $78 \%$ of obese patients experienced $\geq 1$ supratherapeutic aPTT value versus $83 \%$ of nonobese patients.

The number of IV UFH dose adjustments required to achieve a therapeutic aPTT ranged between 0 and 6 with an average of 2 adjustments per patient in both groups (Table 2).

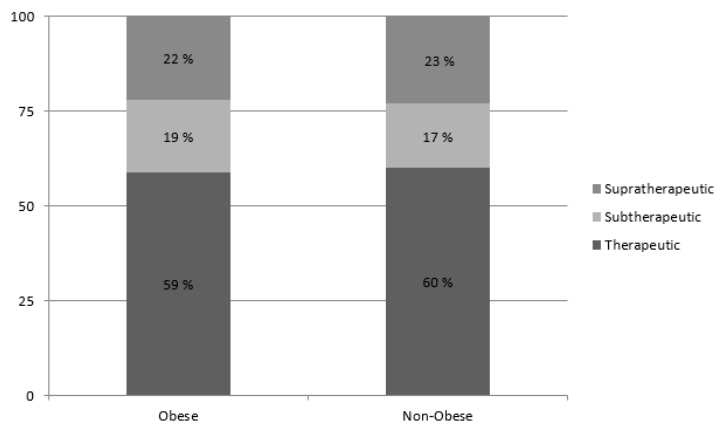

Figure 1: aPTT values within first $24 \mathrm{~h}$ of IV unfractionated heparin initiation

Table 2: Number of unfractionated heparin dose adjustments required to achieve a therapeutic aPTT

\begin{tabular}{lcc}
\hline Variable & Obese group & $\begin{array}{c}\text { Non- obese } \\
\text { group }\end{array}$ \\
\hline Average per patient & 2 & 2 \\
Range per group & $0-6$ & $0-5$ \\
\hline
\end{tabular}

A total of six patients experienced bleeding (two in the obese group and four in the non-obese group). In the obese group, one patient experienced a minor nosebleed and one experienced minor hematochezia. Both were considered to be critically ill due to other complications (one with metastatic cancer and the other with cardiac complications). In the nonobese group, two patients experienced gastrointestinal bleeding (which led to discontinuation of therapy), and two patients experienced hematochezia. There were no thrombotic events in either group.

\section{DISCUSSION}

We found equivalent outcomes between the AjBW-based dosing of UFH in obese patients and the standard ABW-based dosing of UFH in non-obese patients. The results did not show statistically significant difference in achieving a therapeutic aPTT within the first $24 \mathrm{~h}$ while dosing UFH using AjBW in obese patients or $A B W$ non-obese patients.

These results were similar to the findings of Fan et al [11]. They found that about $57 \%$ of obese patients who received AjBW-based dosing and $51 \%$ of non-obese patients who received ABWbased dosing achieved a therapeutic aPTT within the first $24 \mathrm{~h}$ of UFH initiation with no statistically significant difference. Both studies defined obesity as an ABW greater than $130 \%$ of IBW. Furthermore, Barletta et al studied ABW-based nomogram for UFH dosing in morbidly obese 
(BMI > $40 \mathrm{~kg} / \mathrm{m}^{2}$ ) and non-obese patients and found that morbidly obese patients experienced significantly higher mean aPTT values versus non-obese patients at both $6 \mathrm{~h}(155 \mathrm{vs} .135 \mathrm{~s}, p$ $=0.02)$ and $12 \mathrm{~h}$ (141 vs. $117 \mathrm{~s}, p=0.012)$ [10].

On the other hand, Yee and Norton found that the mean value of the first aPTT was lower when using AjBW-based dosing in obese patients than ABW-based dosing in non-obese patients ( $p$ $<0.05$ ). And thus, they concluded that $A B W$ is more suitable for dosing UFH in obese patients; however, they categorized obese patients as those with $>10 \mathrm{~kg}$ above IBW which may have allowed the inclusion of patients that most studies would not have defined as obese [8].

Another study compared the use of ABW-based dosing of UFH in obese $(n=20)$ and non-obese $(n=20)$ patients with obesity defined as an ABW greater than $30 \%$ of IBW [7]. They found that 45 $\%$ of obese and $60 \%$ of non-obese patients achieved a therapeutic aPTT within the first $24 \mathrm{~h}$. Nevertheless, they reported that $45 \%$ of obese and $35 \%$ of non-obese patients exceeded the therapeutic aPTT range, while our study only showed $22 \%$ of obese patients and $23 \%$ of nonobese patients exceeded therapeutic aPTT ranges.

\section{Limitations of the study}

The main limitations of the study include the small sample size used and the retrospective design.

\section{CONCLUSION}

Utilizing AjBW-based dosing of UFH in obese patients is effective as the standard ABW-based dosing of UFH in non-obese patients for the treatment of VTE. However, further prospective studies utilizing a standard definition of obesity and comparing AjBW-based vs ABW-based dosing in obese patients are warranted.

\section{DECLARATIONS}

\section{Acknowledgement}

We would like to thank the Deanship of Scientific Research at Umm Al-Qura University for supporting this work via Grant Code: 19-MED-101-0044.

\section{Conflict of interest}

The authors declare that they have no known competing financial interests or personal relationships that could have appeared to influence the work reported in this paper.

\section{Contribution of authors}

We declare that this work was done by the authors named in this article and all liabilities pertaining to claims relating to the content of this article will be borne by the authors. Mohammed Alessa, Lori Hornsby and Abdullah A Alhifany designed the study, extracted, reviewed, and analyzed the data and were major contributors in writing manuscript. Jawaher Gramish, Hind Almodaimegh and Moteb A Khobrani reviewed the analysis and contributed in writing and reviewing the manuscript.

\section{Open Access}

This is an Open Access article that uses a funding model which does not charge readers or their institutions for access and distributed under the terms of the Creative Commons Attribution License (http://creativecommons.org/licenses/by/ 4.0) and the Budapest Open Access Initiative (http://www.budapestopenaccessinitiative.org/rea d), which permit unrestricted use, distribution, and reproduction in any medium, provided the original work is properly credited.

\section{REFERENCES}

1. Glaser A, Arakaki D, Chan GM, Hoffman RS. Randomised trial of intranasal versus intramuscular naloxone in prehospital treatment for suspected opioid overdose. Med J Aust, 2005. 182(8): p. 427; author reply 427, 429.

2. Ageno $W$, Becattini $C$, Brighton $T$, Selby $R$, Kamphuisen $P W$. Cardiovascular risk factors and venous thromboembolism: a meta-analysis. Circulation, 2008; 117(1): 93-102.

3. Hanley MJ, Abernethy DR, Greenblatt DJ. Effect of obesity on the pharmacokinetics of drugs in humans. Clin Pharmacokinet, 2010. 49(2): 71-87.

4. Hurewitz AN, Khan SU, Groth ML, Patrick PA, Brand DA. Dosing of unfractionated heparin in obese patients with venous thromboembolism. J Gen Intern Med, 2011. 26(5): 487-491.

5. Jain R, Chung SM, Jain L, Khurana M, Lau SW, Lee JE, Vaidyanathan J, Zadezensky I, Choe S, Sahajwalla CG. Implications of obesity for drug therapy: limitations and challenges. Clin Pharmacol Ther, 2011; 90(1): 77-89.

6. Raschke RA, Reilly BM, Guidry JR, Fontana J R, Srinivas $S$. The weight-based heparin dosing nomogram compared with a "standard care" nomogram. A randomized controlled trial. Ann Intern Med 1993; 119(9): 874-881. 
7. Spruill WJ, Wade WE, Huckaby WG, Leslie RB. Achievement of anticoagulation by using a weight-based heparin dosing protocol for obese and nonobese patients. Am J Health Syst Pharm 2001. 58(22): 21432146.

8. Yee WP, Norton L, Optimal weight base for a weightbased heparin dosing protocol. Am J Health Syst Pharm 1998; 55(2): 159-162.

9. Hohner EM, Kruer RM, Gilmore VT, Streiff M, Gibbs $H$. Unfractionated heparin dosing for therapeutic anticoagulation in critically ill obese adults. J Crit Care, 2015. 30(2): 395-399.

10. Barletta JF, DeYoung JL. McAllen K, Baker R, Pendleton $K$, Limitations of a standardized weight-based nomogram for heparin dosing in patients with morbid obesity. Surg Obes Relat Dis 2008; 4(6): 748-753.

11. Fan J, John B, Tesdal E, Evaluation of heparin dosing based on adjusted body weight in obese patients. Am J Health Syst Pharm, 2016. 73(19): 1512-1522.
12. Schaefer DC, Hufnagle J, Williams L, Rapid heparin anticoagulation: use of a weight-based nomogram. Am Fam Physician, 1996. 54(8): 2517-2521.

13. Pai MP, Paloucek FP. The origin of the "ideal" body weight equations. Ann Pharmacother, 2000. 34(9): 1066-1069.

14. von Elm E, Altman DG, Egger M, Pocock SJ, Gøtzsche $P C$, Vandenbroucke JP; STROBE Initiative. The Strengthening the Reporting of Observational Studies in Epidemiology (STROBE) statement: guidelines for reporting observational studies. Clin Epidemiol, 2008. 61(4): 344-349

15. Schulman S, Kearon C. Definition of major bleeding in clinical investigations of anti-hemostatic medicinal products in non-surgical patients. J Thromb Haemost, 2005. 3(4): 692-694. 